Check for updates

Cite this: RSC Adv., 2017, 7, 40200

Received 22nd June 2017 Accepted 29th July 2017 DOI: $10.1039 / c 7 r a 06956 a$ rsc.li/rsc-advances

\section{Tuning the electronic and magnetic properties of penta-graphene using a hydrogen atom: a theoretical study $\dagger$}

\author{
Lin-Lin Liu, ${ }^{\text {ab }}$ Ying Wang, ${ }^{\text {ab }}$ Chun-Ping Chen, ${ }^{a b}$ Hong-Xia Yu, ${ }^{\text {ab }}$ Lu-Si Zhao ${ }^{a b}$ \\ and Xiao-Chun Wang (D)*ab
}

Herein, an isolated hydrogen atom absorbed on penta-graphene (PG) was predicted to induce magnetic moments and tune the electronic properties of penta-graphene and was systematically studied using first-principles calculations. The adsorption energy and formation energy calculations suggest that magnetic penta-graphene (M-PG) and weak magnetic penta-graphene (WM-PG) of hydrogen-absorbed $P G$ systems are energetically the most favorable states among the possible hydrogen-absorbed configurations. The hydrogen atom adsorbed on the PG sheet can effectively tune the electronic properties of PG and change it from a semiconductor to half-metallic. In the M-PG system, this spinpolarized state is essentially localized on the three-fold coordinated $\mathrm{C}$ atoms in the first layer opposite to that where the hydrogen atom is chemisorbed. Through changing the bond angle and bond length of the adsorbed hydrogen atom on the PG system, we can remarkably increase the magnetic moment of the hydrogen-absorbed PG system by 137 times. This would allow us to design a magnetic nanoswitch to manipulate the magnetic state of PG. We also obtained the scanning tunneling microscopy (STM) images for future experimental identification. Our findings show that the hydrogen atom absorbed on the PG system will have exciting applications in magnetic storage technology and next-generation electronic and spintronic nanodevices.

\section{Introduction}

Currently, two-dimensional (2D) atomic-layer-based materials are being widely studied due to their atomic scale thickness and unique electronic and magnetic properties..$^{1-3}$ As carbon is durable, light, and abundant, carbon-based magnets are expected to be more stable, versatile, and cheaper than the current transition-metal-based magnets. Contrary to most carbon allotropes with hexagonal building blocks, penta-graphene (PG) is exclusively composed of carbon pentagons. It is predicted that penta-graphene can withstand temperatures as high as $1000 \mathrm{~K}$, has an unusual negative Poisson's ratio and ultrahigh ideal strength, outperforming graphene, and possesses an intrinsic quasi-direct band gap as large as $3.25 \mathrm{eV}{ }^{4}$ Due to these superior properties, penta-graphene is anticipated to be a potential candidate for broad applications in nanoelectronics, nanomechanics, optoelectronics, and photovoltaics. Furthermore, both the mechanical and electronic properties of PG sheets can

aInstitute of Atomic and Molecular Physics, Jilin University, Changchun, 130012, China. E-mail: wangxiaochun@jlu.edu.cn

${ }^{b}$ Jilin Provincial Key Laboratory of Applied Atomic and Molecular Spectroscopy, Jilin University, Changchun, 130012, China

$\dagger$ Electronic supplementary information (ESI) available. See DOI: $10.1039 / \mathrm{c} 7 \mathrm{ra06956a}$ be refined via surface functionalization. $\mathrm{H}$ or $\mathrm{F} \mathrm{sp}^{3}$ functionalization, ${ }^{5,6}$ transition metals, ${ }^{7,8}$ and strain $^{9}$ can be sources of magnetism via the generation of dangling bond (DB) states. Recent theoretical investigations have revealed that hydrogenation can effectively tune the electronic and mechanical properties of penta-graphene. ${ }^{10}$ In addition, the electronic structures of the hydrogenated group IV monolayers are found to be topologically trivial. ${ }^{11}$ Isolated hydrogen atoms absorbed on graphene have been predicted to induce magnetic moments. ${ }^{1}$ J. Zhou et al. have theoretically demonstrated that it is possible to introduce ferromagnetism into graphene via surface modification using hydrogenation. ${ }^{12}$ Thus, we have carried out an in-depth study of hydrogen absorbed on a pentagraphene system.

In this study, using a first-principles theory, we investigated isolated hydrogen atoms absorbed on penta-graphene (PG), which was predicted to induce magnetic moments and tune the electronic property. We defined the two favorable states of isolated hydrogen atom absorbed on PG as the magnetic pentagraphene (M-PG) and the weak magnetic penta-graphene (WM-PG). A new magnetic switch based on on-site proton transfer on the PG system was designed herein. We have also defined the on-site proton transfer as follows: the proton, which is located at the same site of the substrate after transfer, transfers to the other state only with different bond lengths and 
angles between the proton and the substrate. Although the abovementioned on-site proton transfer is quite different from the well-known intermolecular proton transfer and intramolecular proton transfer, it can also obviously change the properties of the system. First, both the binding energy and the formation energy are calculated to characterize the stability of the systems. The on-site proton transfer on the PG system not only changes the electronic band structures, but also changes the magnetic properties of the system. To gain more insight into the electronic structure of the M-PG and WM-PG systems, we calculated the electronic band structures and the spin-polarized local DOS (LDOS). We also calculated the three-dimensional spin polarized charge density to show the magnetic property characteristics of the systems. The charge density difference and Bader charge analyses can help to predict the electron transfer between the $\mathrm{H}$ atom and the PG sheet. The study presented herein suggests that the hydrogen-absorbed PG system can be a promising magnetic nanoswitch. Scanning tunneling microscopy (STM) images are demonstrated to reveal the relationship between the configurations of atoms and spatial distributions of electronic states related to PG adsorbed by hydrogen atom.

\section{Computational methods}

Spin-polarized density functional theory (DFT) calculations have been performed using the VASP code, which is based on the Perdew-Burke-Ernzerhof (PBE) version of the generalized gradient approximation ${ }^{\mathbf{1 3}}$ and the projector augmented wave representation, ${ }^{\mathbf{1 4 , 1 5}}$ including spin polarization corrections and dipole moment corrections. ${ }^{\mathbf{1 6 , 1 7}}$ The dipole moment corrections were employed to account for the possible dipole moments in the hydrogen atom absorbed on the penta-graphene system.18,19 The plane-wave cut-off energy of $520 \mathrm{eV}$ was used for all the elements in the calculations. PG is represented using a unit cell containing 6 atoms, with a $(1 \times 1)$ structure in the $x-y$ plane and a vacuum layer of $15 \AA$ in the direction perpendicular to the PG sheet to maintain low interactions between periodic images. The first Brillouin zone was sampled using the $11 \times 11 \times 1$ Monkhorst-Pack $k$-point mesh; Gaussian smearing with a width of $\sigma=0.02 \mathrm{eV}$ was used in all the calculations. In the energy minimization, we allowed all atoms in the slab to fully relax. Energy convergence was reached when the forces on the relaxed atoms were less than $0.02 \mathrm{eV} \AA^{-1}$.

For the calculation of the STM images, the Tersoff-Hamann approximation was used. ${ }^{20}$ Although it is expressed in a simple form, the method is widely used and well known to be valid for many systems. ${ }^{21-23}$ In this system, the tunneling current is proportional to the surface local density of states (LDOS) at the tip position integrated over an energy range restricted by the applied bias voltage. Hence, the STM images are generated from the isosurface of the spatial distribution formed by the integration of the LDOS over the energy range from the Fermi energy $E_{\mathrm{Fermi}}$ to $E_{\mathrm{Fermi}}-\mathrm{eV}$ with an applied voltage $V$, i.e., $\int_{0}^{\mathrm{eV}} \rho\left(x, y, z ; E_{\mathrm{F}}-\mathrm{eV}+\varepsilon\right) \mathrm{d} \varepsilon$. Thereby, the calculated STM images should correspond to experimental constant-current images.

\section{Results and discussion}

Before discussing the hydrogen atom absorbed on the PG system, we have demonstrated the optimized pristine PG sheet. Unlike graphene where all carbon atoms are $\mathrm{sp}^{2}$-hybridized, there are two chemically nonequivalent carbon atoms in PG. The unit cell contains two four-fold coordinated and four threefold coordinated carbon atoms. For the convenience of discussion, we grouped the four-fold and three-fold coordinated carbon atoms of the pristine $\mathrm{PG}$ sheet as $\mathrm{C}_{1}$ and $\mathrm{C}_{2}$, respectively, as shown in Fig. 1. Herein, the calculated lattice constant of the pristine PG sheet is $3.64 \AA$, which is in good agreement with the theoretical value of $3.64 \AA_{;} ;$this indicates the accuracy of our computational approaches.

We then focused on the hydrogen atom absorbed on the PG system. Due to the special atomic configuration, Xiaoyin Li et al. have found that only the three-fold coordinated carbon atoms can adsorb hydrogen atom to form the hydrogenated PG sheet. ${ }^{\mathbf{1 0}}$ As shown in Fig. 1, after the hydrogen atom was absorbed on the PG sheet, one of the three-fold coordinated carbons became four-fold coordinated. Moreover, we grouped the three-fold coordinated carbon atoms of the hydrogen-absorbed PG sheet as $\mathrm{C}_{3}$ and labelled the three bonds associated with the $\mathrm{C}_{3}$ atom as $\mathrm{d}_{1}, \mathrm{~d}_{2}$, and $\mathrm{d}_{3}$ (see Fig. 1).

First, the hydrogen atom adsorbed on the PG sheet was studied. The optimized structure parameters of the M-PG and WM-PG systems are shown in Fig. 2. Compared to those for the PG sheet, ${ }^{4}$ the bond lengths of $\mathrm{d}_{1}, \mathrm{~d}_{2}$, and $\mathrm{d}_{3}$ increased from $1.34 \AA$, $1.55 \AA$, and $1.55 \AA$ to 1.48/1.46 ̊, 1.62/1.66 ̊, and 1.62/ $1.66 \AA$ for the M-PG/WM-PG system, respectively, due to the adsorption of hydrogen; this implies the transformation of the

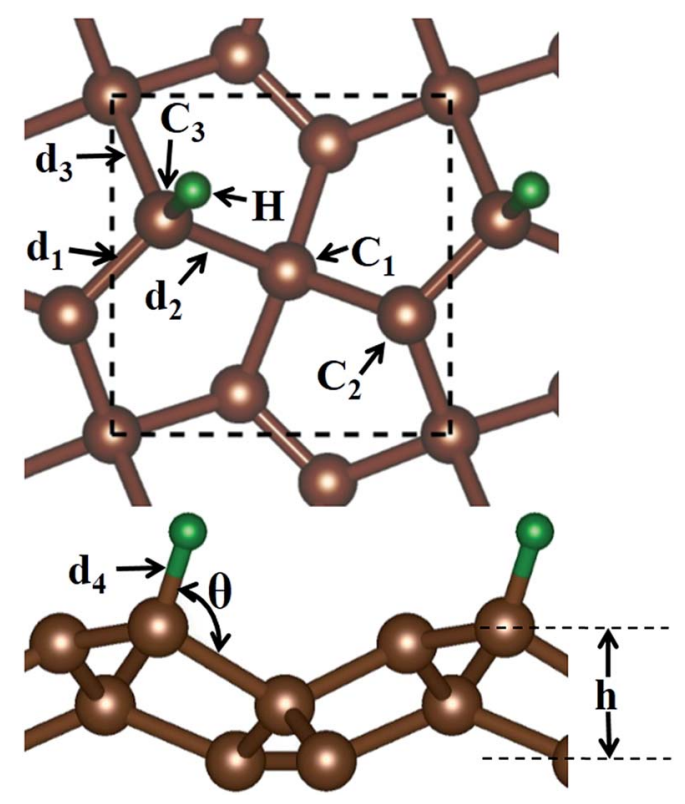

Fig. 1 Top and side views of the optimized structure of the hydrogen atom adsorbed on the penta-graphene sheet. The square marked by black dashed lines denotes the unit cell. Carbon and hydrogen atoms are denoted with brown and green balls, respectively. 


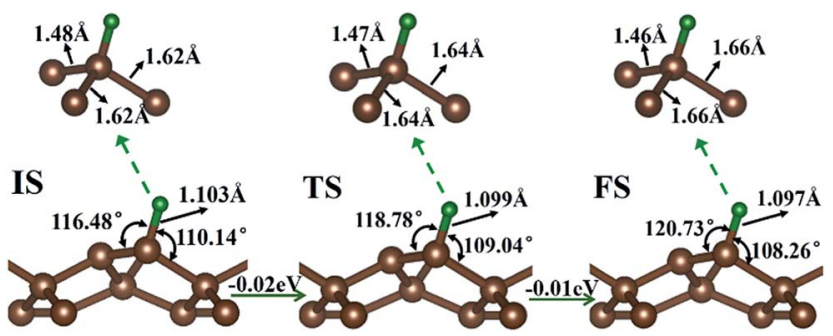

Fig. 2 Atomic configurations and the relevant structural parameters of the IS, TS, and FS for the whole process of on-site proton transfer on the penta-graphene system from the M-PG to L-PG system. The energy changes between neighboring states are displayed. Carbon and hydrogen atoms are denoted with brown and green balls, respectively.

double bond between $\mathrm{C}_{2}$ and $\mathrm{C}_{3}$ atoms into a single bond. In addition, the surface modification of PG elongates the bond length of $\mathrm{d}_{1}, \mathrm{~d}_{2}$, and $\mathrm{d}_{3}$, thus partially releasing the strain stored in the pristine structure. According to these model studies, ${ }^{24-26}$ the changes in the bond lengths would lead to increased electron localization and splitting of the spin states. We defined $h$ as the vertical coordinate difference of the top and bottom carbon layers (see Fig. 1). It can be observed that after the adsorption of hydrogen, the $h$ increases to 1.402/1.399 $\AA$ of the M-PG/WM-PG system from $1.206 \AA$ of the PG sheet. Through the on-site proton transfer on the PG sheet, the hydrogen adsorption position on PG did not change, but the angle $(\theta)$ significantly changed.

Both the binding energy $E_{\mathrm{B}}$ and the formation energy $E_{\mathrm{F}}$ were introduced to characterize the stability of the M-PG and WM-PG systems. Following the method reported in previous studies, ${ }^{27-29}$ the binding energy and the formation energy of the hydrogen atom absorbed on the PG system were calculated using the following formula:

$$
\begin{gathered}
E_{\mathrm{B}}=E_{\mathrm{PG}}+E_{\mathrm{H}}-E_{\mathrm{Total}}, \\
E_{\mathrm{F}}=E_{\mathrm{Total}}-\left(E_{\mathrm{PG}}+E_{\mathrm{H}_{2}} / 2\right),
\end{gathered}
$$

where $E_{\text {Total }}, E_{\mathrm{PG}}, E_{\mathrm{H}}$, and $E_{\mathrm{H}_{2}}$ stand for the total energy of the ground state configuration of the M-PG/WM-PG system, pristine $\mathrm{PG}$, and isolated hydrogen atom and the total energy of the free $\mathrm{H}_{2}$ molecule, respectively. ${ }^{30}$ The binding energy represents the strength of the $\mathrm{C}-\mathrm{H}$ covalent bond, and in this definition, the larger the absolute value, the stronger the $\mathrm{C}-\mathrm{H}$ covalent bond. The formation energy can be regarded as a measure of the stability against molecular desorption from the PG surface. In this definition, a positive (negative) value means the process is endothermic (exothermic). That is to say, the state of hydrogen absorbed on the PG system has a higher (lower) energy than the state of pristine PG and $\mathrm{H}_{2}$ molecules. If we obtain a positive (negative) formation energy value, it means that the system of hydrogenated graphene is unstable (stable). Therefore, the binding energy and formation energy indicate the stability of hydrogen adsorbed on the PG system. The calculated binding energies are $2.77 \mathrm{eV}$ and $2.74 \mathrm{eV}$ for the M-PG and WM-PG systems, respectively, which are larger than $\sim 1.13 \mathrm{eV}$ for graphene. ${ }^{28}$ This is because the $\mathrm{C}_{3}-\mathrm{H}$ bond lengths are $1.103 \AA$ and $1.097 \AA$ for the M-PG and WM-PG systems, respectively, which are smaller than $1.132 \AA$ for graphene. ${ }^{31}$ The larger binding energy and smaller bond length indicate that the attractive interactions between the hydrogen atoms and the PG sheet are strong. Then, after several state changes between the M-PG and WM-PG states, the hydrogen atoms can still be stably adsorbed on the PG sheet. The possible reason is that the adsorption of hydrogen atom on PG enlarges the bond length between $\mathrm{C}_{3}$ and the neighbouring $\mathrm{C}$ atoms; this partially decreases the interactions between them. This can be understood from the following facts: the buckling structure of PG has more space for the adsorbed $\mathrm{H}$ atom, and the three-fold coordinated $\mathrm{C}_{3}$ atom in $\mathrm{PG}$ are chemically more active than the $\mathrm{C}$ atoms in graphene that are relatively inert due to the delocalized $\pi$ electrons. ${ }^{28}$ We have calculated that the formation energies of the M-PG and WM-PG systems are $-0.60 \mathrm{eV}$ and $-0.64 \mathrm{eV}$, respectively. The negative formation energies herein indicate that the $\mathrm{H}$ atom is energetically favorable to be adsorbed on the PG sheet.

Proton and $\mathrm{H}$ atom transfer mechanisms and dynamics represent one of the most fundamental processes involved in chemical reactions as well as in living systems. ${ }^{32-34}$ Through a spin-polarized calculation, we found that for the M-PG system, the total energy of antiferromagnetic (AFM) state was lower than that of the ferromagnetic (FM) state by $0.13 \mathrm{eV}$. For WM-PG, the total energy of the antiferromagnetic (AFM) state was lower than that of the ferromagnetic (FM) state by $0.02 \mathrm{eV}$, whereas the highest magnetic moment of a single atom was still very low $\left(0.003 \mu_{\mathrm{B}}\right)$. The local configurations and the relevant structural parameters of each state for the whole on-site proton transfer on PG are displayed in Fig. 2. The M-PG and the WM-PG systems were selected as the IS and FS, respectively. At the TS, the lengths of $d_{3}$ and $d_{4}$ are shorter than those in the IS, and the $\theta$ also becomes smaller than that in the IS. However, the lengths of $d_{1}$ and $d_{2}$ are shorter than those in the IS. The reaction energy for this step is $0.02 \mathrm{eV}$, and the $\mathrm{H}$ binding energy for the IS with respect to the $\mathrm{PG}$ sheet is $2.77 \mathrm{eV}$, suggesting that the process should be energetically favorable. The obtained results show that the change in energy for the on-site proton transfer on PG is $0.03 \mathrm{eV}$. This energy change is caused by the electronic organization change associated with the atomic configuration change of the hydrogen atom adsorbed on the PG systems. UVinduced intramolecular proton transfer reactions have been observed and reported for numerous compounds. ${ }^{35}$

The electronic band structures for the M-PG and WM-PG systems are depicted in Fig. 3. The Fermi level is set at $0 \mathrm{eV}$. The electronic band structures of the M-PG and WM-PG systems are different. Interestingly, the M-PG system presents a halfmetallic characteristic with the spin up channel being metallic and the spin down channel being semiconducting, as shown in Fig. 3(a). It can be seen that this M-PG system demonstrates magnetic half-metallicity with a total magnetic moment of $0.551 \mu_{\mathrm{B}}$ per unit cell. As plotted in Fig. 3(b), the paired spin-up channel (semiconducting) and the spin-down channel (metallic) of the band structures imply that the WMPG system has very small magnetic characteristic with a total 

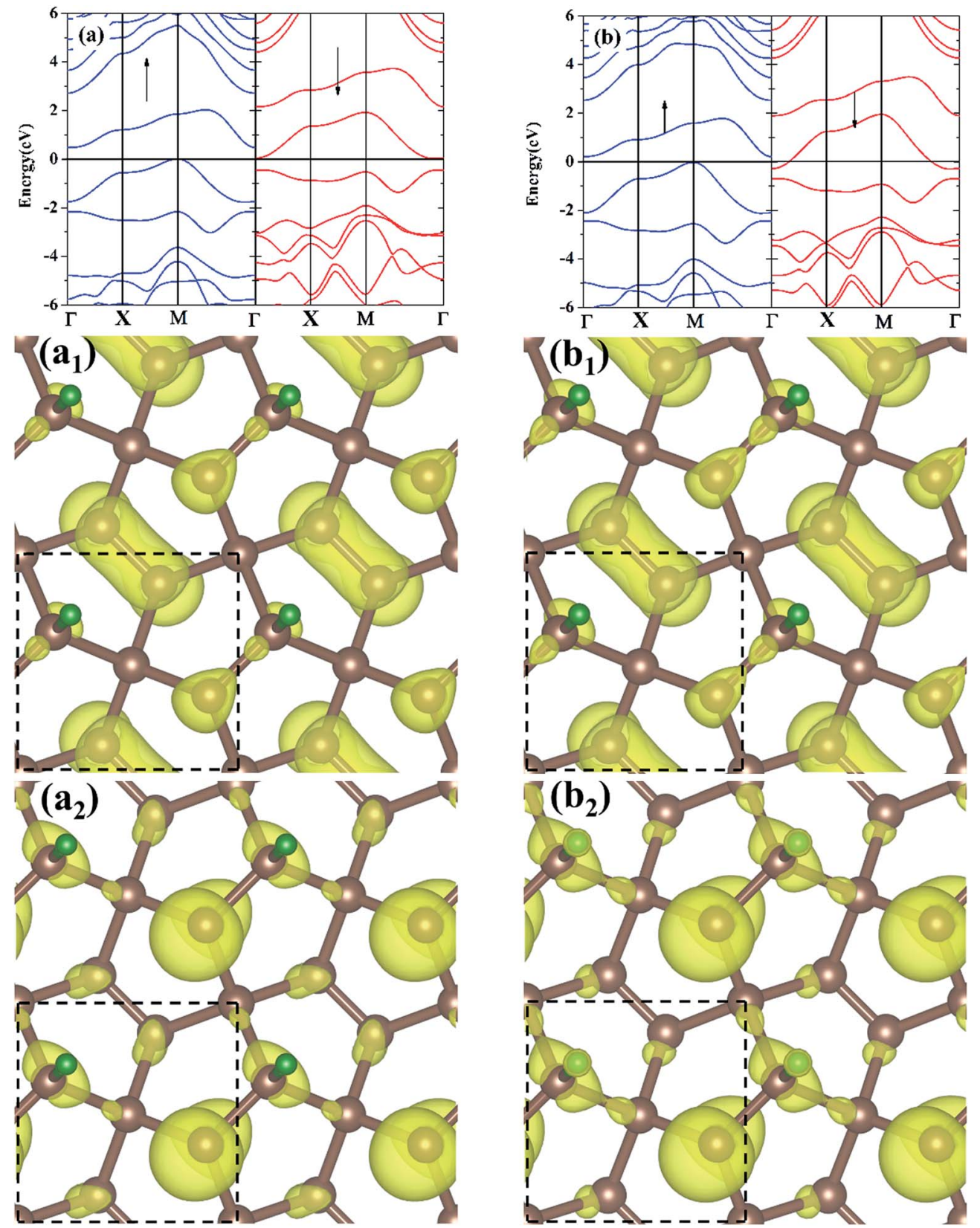

Fig. 3 Electronic band structure of the M-PG (a) and L-PG (b) systems. The arrow denotes the spin-polarized direction. The Fermi level is shifted to $0 \mathrm{eV}$. The band-decomposed charge density distributions of the M-PG $\left(a_{1}\right)$ and $\left(a_{2}\right)$ and L-PG $\left(b_{1}\right)$ and $\left(b_{2}\right)$. $\left(a_{1}\right)$ and $\left(b_{1}\right)$ are the highest occupied bands, and $\left(a_{2}\right)$ and $\left(b_{2}\right)$ are the lowest unoccupied bands. Yellow isosurface represents spin density $\left(+0.015\right.$ e bohr $\left.{ }^{-3}\right)$. The square marked by black dash lines denotes the unit cell. Carbon and hydrogen atoms are denoted with brown and green balls, respectively.

magnetic moment of $0.004 \mu_{\mathrm{B}}$ per unit cell. The valence band maximum (VBM) and conduction band minimum (CBM) are located at the same $k$ points in the momentum space in the spin-up electronic band structure for the M-PG and WM-PG systems, but the CBM is located at $0.482 \mathrm{eV}$ in the M-PG system, which is higher than $0.268 \mathrm{eV}$ in the WM-PG system. Moreover, the on-site proton transfer has much effect on the band structures with the spin-polarized direction of the hydrogen-adsorbed PG system. The PG sheet possesses an intrinsic quasi-direct band gap as large as $3.25 \mathrm{eV} .{ }^{4}$ As a result of the $\mathrm{H}$ atom adsorption on the PG sheet, a new band originating from the $\mathrm{H}$ atom above the Fermi energy and the electronic bands of the PG sheet are modified, according to the electronic band structure of PG shown in Fig. S1 in ESI. $\dagger$ This can be attributed to the redistribution of charge density. The charge density distributions of the band edge states for the M-PG and WM-PG systems were calculated as well for comparison, and the results are presented in Fig. $3\left(a_{1}\right),\left(a_{2}\right),\left(b_{1}\right)$, and $\left(b_{2}\right)$. There is more charge accumulation on the $\mathrm{H}$ atom of the WM-PG system (Fig. 3( $\left.\mathrm{b}_{2}\right)$ ) than that in the M-PG system (Fig. 3( $\left.\mathrm{a}_{2}\right)$ ). Compared to the band-decomposed charge density distribution of the PG sheet, ${ }^{4}$ the charge density distributions of the band edge states 
for the M-PG and WM-PG systems are different. This is because the adsorption of $\mathrm{H}$ atom locally removes the $\mathrm{p}_{z}$ orbitals from the $\mathrm{C}_{3}$ atom; this results in full electron delocalization and changes the charge distribution of the $\mathrm{C}_{2}-\mathrm{C}_{3}$ bonds. Thus, the adsorption of $\mathrm{H}$ atom gives rise to disappearance of the band gap near the Fermi level for the M-PG and WM-PG systems.

To gain more insight into the electronic structure of the hydrogen atom absorbed on the PG system, the spin-polarized local DOS (LDOS) of the M-PG and WM-PG systems were studied. The Fermi level is set at $0 \mathrm{eV}$, as shown in Fig. 4(b) and (d). We have found that the $\mathrm{H}$ atom is considered to form chemical bonds with the $\mathrm{C}_{3}$ atom in the two systems. The electronic structure of $\mathrm{PG}$ affected by $\mathrm{H}$ adsorption is reflected in the appearance of the electronic state at $E_{\mathrm{F}}$ as compared to the LDOS of PG in Fig. S2 in ESI. $\uparrow$ Therefore, it was instructive that we investigated the binding mechanisms in detail. First, the M-PG system is considered. The $\mathrm{C}$ atom $\left(2 \mathrm{~s}^{2} 2 \mathrm{p}^{2}\right)$ has four valence electrons; three of them form the $\mathrm{C}-\mathrm{C}$ covalent $\sigma$ bonds, whereas the fourth forms covalent $\sigma$ bond with the $\mathrm{H}$ atom, which results in very high binding energy. Atomic $\mathrm{H}$ chemisorbs on the PG sheet on top of the $\mathrm{C}_{3}$ atom, changing the initial $\mathrm{sp}^{2}$ hybridization of carbon to essentially $\mathrm{sp}^{3}$ and effectively removing the corresponding $\mathrm{p}_{z}$ orbital, ${ }^{36}$ creating a threedimensional network of $\mathrm{sp}^{2}$ and $\mathrm{sp}^{3}$ carbon atoms. This approach demonstrates the possibility for the mixture of $\mathrm{sp}^{2}-$ $\mathrm{sp}^{3}$ bonding and the magnetic coupling between $\pi$-electrons to induce magnetism into the PG sheet. ${ }^{37-40}$ As shown in Fig. 4(b), there are partially occupied $\mathrm{C}_{2} 2 \mathrm{p}$ states near the Fermi level for the M-PG system. Moreover, the spin-up and spin-down DOS of the $\mathrm{C}_{1}, \mathrm{C}_{3}, \mathrm{C}_{2}$, and $\mathrm{H}$ orbitals and the total DOS are asymmetric (especially near the Fermi level); this leads to a magnetic moment of $0.551 \mu_{\mathrm{B}}$ per unit cell. Thus, our results stress the importance of hydrogen for the development of the magnetic state, and hydrogen can play a decisive role. ${ }^{41}$ The analysis of the spin density for the M-PG system shows that it is highly localized on the $\mathrm{C}_{2}$ atom. As shown in Fig. 4(a), the spin polarized charge distribution is in a wide area. Namely, besides the $\mathrm{C}_{2}$ atom, the $\mathrm{C}_{3}$ and $\mathrm{H}$ atoms also contribute to produce the magnetic properties. ${ }^{1}$ For the WM-PG system, the electronic structure is different. Through the on-site proton transfer, the
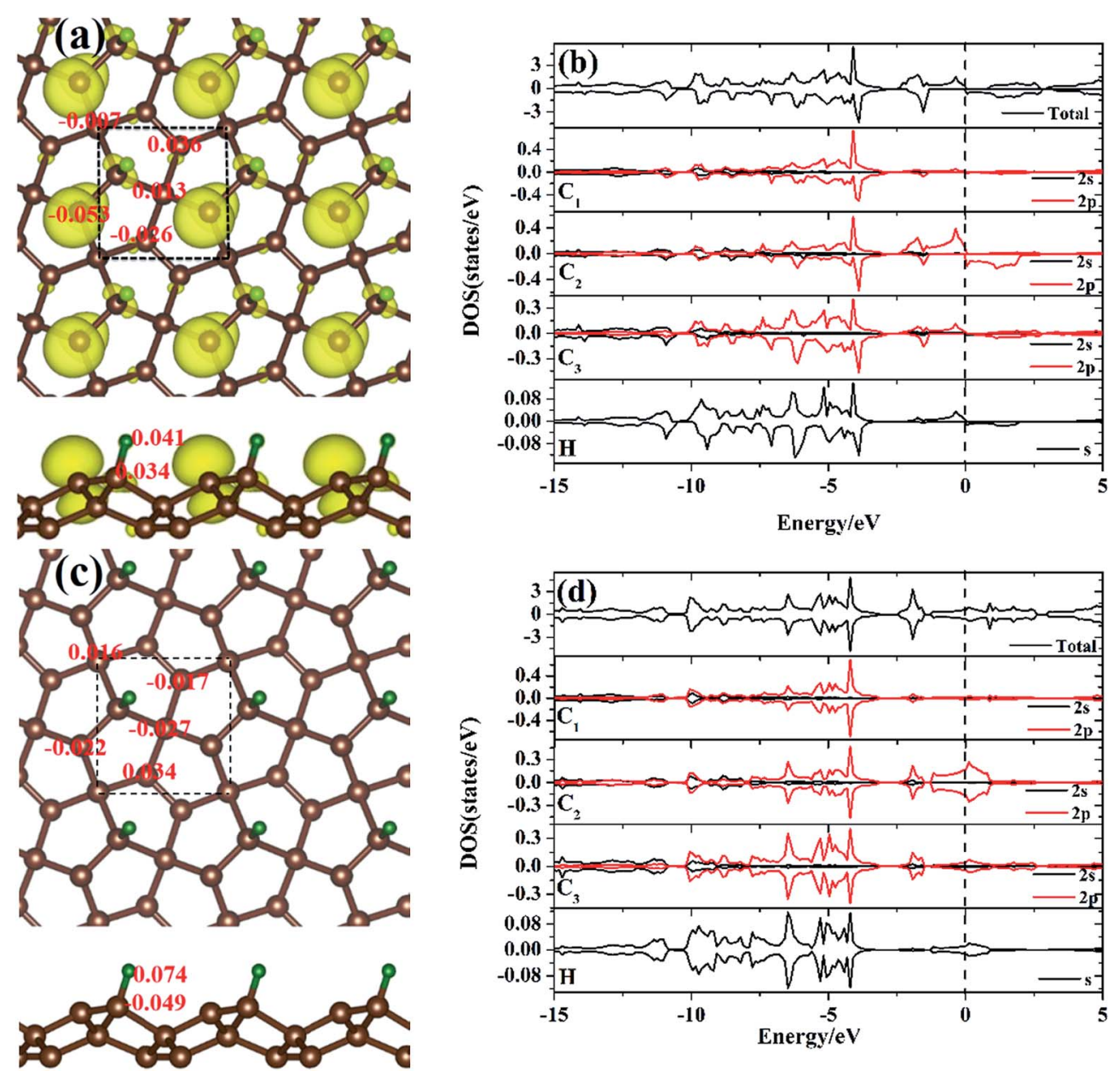

Fig. 4 Charge density distribution in the three-dimensional spin polarized charge density map of the M-PG and L-PG systems combining the Bader charge analysis are shown in (a) and (c), respectively. Yellow isosurface represents spin density $\left(+0.009\right.$ e bohr $\left.{ }^{-3}\right)$. The squares marked by black dashed lines denote the unit cells. The spin-polarized LDOS of the M-PG and L-PG systems are shown in (b) and (d), respectively. The positive and negative LDOS denote the spin-up and spin-down states, respectively. The vertical dashed line indicates the Fermi level. Carbon and hydrogen atoms are denoted with brown and green balls, respectively. 
proton $\mathrm{H}$ is still chemically adsorbed on the PG sheet on top of the same $\mathrm{C}_{3}$ atom with different bond lengths and angles. As shown in Fig. $4(\mathrm{~d})$, the $\mathrm{C}_{1}, \mathrm{C}_{2}$, and $\mathrm{C}_{3} 2 \mathrm{p}$ orbitals and $\mathrm{H} \mathrm{s}$ orbitals significantly contribute to the total DOS between approximate -15 and $5 \mathrm{eV}$, and the presence of $\mathrm{H}$ s orbital peaks at the Fermi level further signifies the hybridization between the $\mathrm{H}$ atom and the $\mathrm{C}_{3}$ atom. The electronic states near the Fermi level not only originate from the $\mathrm{sp}^{2}$ hybridized $\mathrm{C}_{2}$ atoms, but also originate from the $\mathrm{sp}^{3}$ hybridized $\mathrm{C}_{3}$ atoms; this is further confirmed by calculating the band-decomposed charge density distributions (Fig. 3( $\left.b_{1}\right)$ and $\left(b_{2}\right)$ ). Unlike those in the M-PG system, the spinup and spin-down DOS of the $\mathrm{C}_{1}, \mathrm{C}_{2}$, and $\mathrm{C}_{3}$ orbitals and the total DOS (Fig. 4(d)) were symmetric and induced very weak magnetic moments $\left(0.004 \mu_{\mathrm{B}}\right.$ per unit cell) because the coupling between the on-site proton transfer and the electron transfer resulted in the quenching of the spin magnetic moments; this was in good agreement with the analysis of the spin density for the WM-PG system (Fig. 4(c)). Because of the big difference in the $\mathrm{C}_{2}$ atom electronic configurations between the M-PG and WM-PG systems, the electrons in the $\mathrm{C}_{2}$ atom of the M-PG system are easier to be polarized. The hydrogen atoms with different on-site energy states on penta-graphene not only change the band structures but also change the magnetism of the carbon atoms in the PG. H. Gonzalez-Herrero's work shows that for large enough electron (hole) doping, the H-induced magnetic state changes to a single nonmagnetic state for the isolated hydrogen atoms absorbed on a graphene system. ${ }^{1}$ Contrary to H. Gonzalez-Herrero's work, herein, for PG with the hydrogen atoms absorbed on it, the mechanical movement of the absorbed hydrogen atoms can realize the switch between the magnetic state and the weak magnetic state without charge doping. This on-site proton transfer on the PG system could be used in a magnetic nanoswitch and magnetic storage technology.

To quantify the charge distribution, we performed Bader charge analysis, ${ }^{42}$ which helped to show the charge transfer (a)
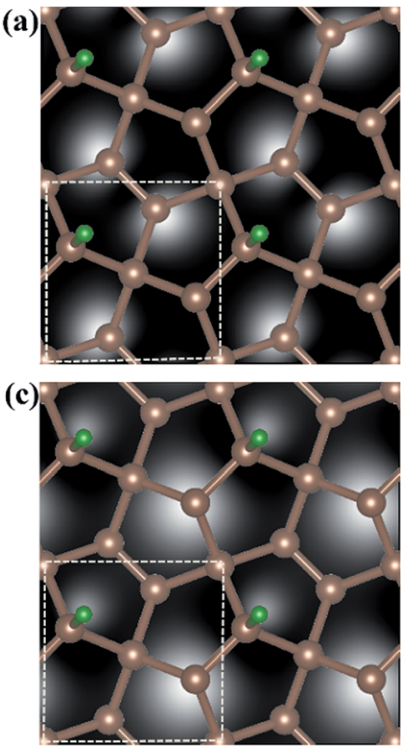
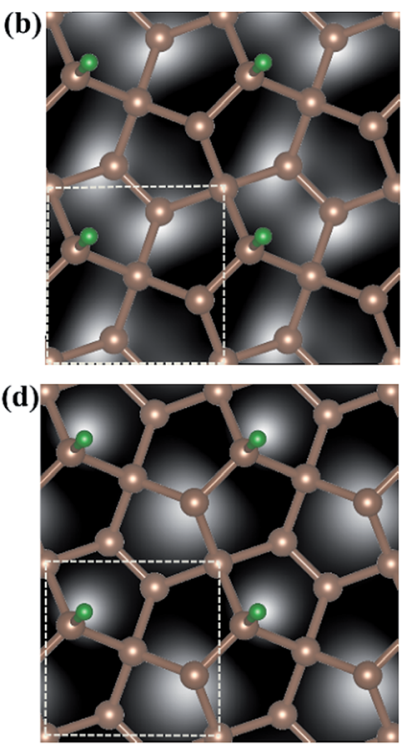

Fig. 6 Computed constant-height STM images of the $\mathrm{H}$ atom adsorbed on the PG sheet calculated with a negative tip potential of $-1 \mathrm{eV}$ : (a) and (c) show the M-PG system; (b) and (d) show the L-PG system. The maximal height of tip in the STM images is set to be approximately $1.5 \AA$ under the third C layer in penta-graphene sheet in the (a) and (b). The maximal height of tip in the STM images is set to be about $0.5 \AA$ above the adsorbed $\mathrm{H}$ atom on penta-graphene sheet in the (c) and (d). The dashed lines show the unit cell. Carbon and hydrogen atoms are denoted with brown and green balls, respectively.

values for the $\mathrm{H}$ atom and the PG sheet, as depicted in Fig. 4(a) and (c). The charge density difference $(\Delta \rho(r))$, which helps to predict the electron transfer between the $\mathrm{H}$ atom and the PG sheet, is defined as $\Delta \rho(r)=\rho(\mathrm{H}+\mathrm{PG})-\rho(\mathrm{PG})-\rho(\mathrm{H})$, where $\rho(\mathrm{H}$ $+\mathrm{PG})$ is the charge density of the M-PG or WM-PG system and $\rho(\mathrm{PG})$ and $\rho(\mathrm{H})$ are the charge densities of the independent PG sheet and the $\mathrm{H}$ atom, respectively, which are separately
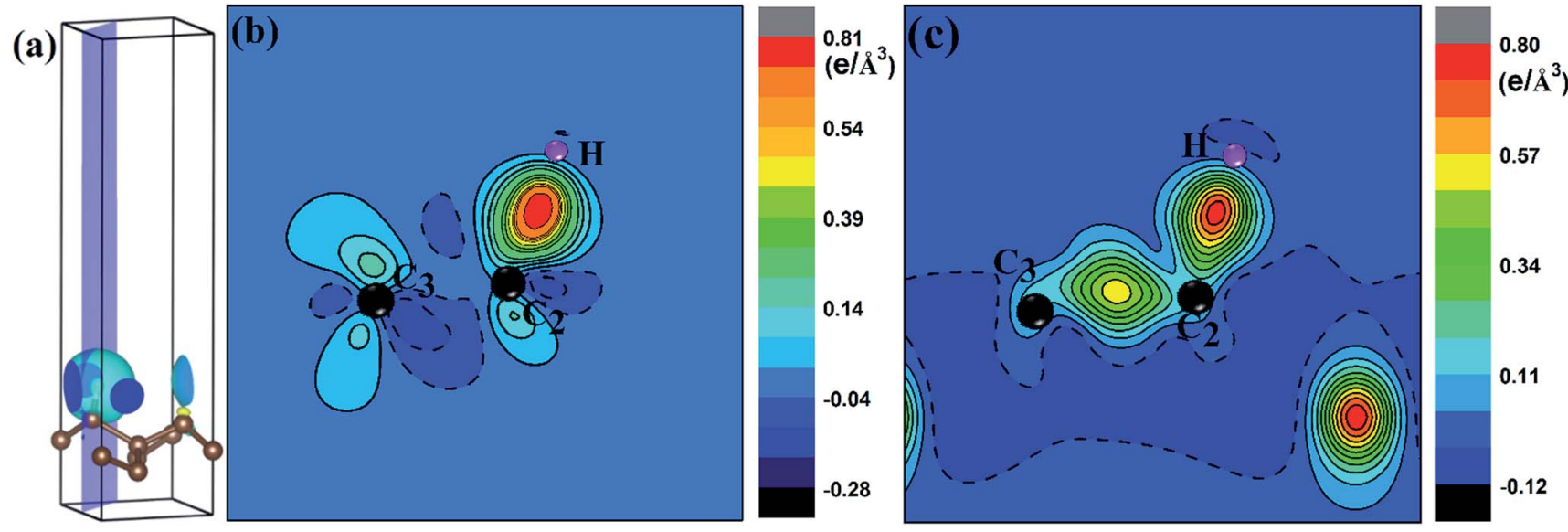

Fig. 5 The charge density difference (see text for the definition) for $\mathrm{H}$ adsorbed on PG sheet. (a) shows the 3D plots of charge density difference of $\mathrm{H}$ absorbed on the PG sheet. (b) and (c) are corresponding 2D plots on the blue plane in (a) for M-PG and L-PG systems, respectively. The right labels of the 2D plots show the contour value of charge density difference. A positive value (solid contour line) indicates an increase in electron density and a negative value (dashed contour line) means an electron density loss with respect to the superposition of the atomic electron density. 
calculated using the same atomic coordinates and supercell as those in the corresponding M-PG or WM-PG system. The Bader charge analyses of the M-PG system (Fig. 4(c)) shows that $\mathrm{C}_{3}$ and $\mathrm{H}$ atoms are positively charged, and the two $\mathrm{C}$ atoms neighboring to the $\mathrm{C}_{3}$ atom are negatively charged, indicating that the unpaired electron on the $\mathrm{H}$ atom is transferred to the PG sheet and the $\mathrm{C}_{3}-\mathrm{H}$ bond is polarized; this is in good agreement with the electron density difference plotted in Fig. 5(b). Charge was obviously accumulated in the region between the $\mathrm{C}_{3}$ and $\mathrm{H}$ atoms, which indicates that the covalent bond formed between the adsorbed $\mathrm{H}$ and the PG sheet. This fact plays a role in the stabilization of the M-PG system, as discussed above. As for the WM-PG system, the Bader charge analyses (Fig. 4(a)) shows that two $\mathrm{C}$ atoms neighboring to the $\mathrm{C}_{3}$ atom are negatively charged. This can be attributed to the adsorption of the $\mathrm{H}$ atom. The negatively charged $\mathrm{C}_{3}$ atom pulls the positively charged $\mathrm{H}$ atom towards the surface. The $\mathrm{H}$ atom very strongly interacts with the $\mathrm{C}_{3}$ atom of the WM-PG system, and the $\mathrm{C}_{3}$ atom traps electrons from the $\mathrm{H}$ atom and become negatively charged. As shown in Fig. 5(c), there is not only a significant charge accumulation between the $\mathrm{C}_{2}$ and $\mathrm{C}_{3}$ atoms, but also a large charge accumulation between the $\mathrm{C}_{3}$ and $\mathrm{H}$ atoms. Thus, there are certain charge transfers from $\mathrm{H}$ atom to $\mathrm{C}_{3}$ atom area. Basically, the change trend of the charge transfer for the WM-PG system is in good agreement with that of the element electronegativity. According to the Pauling scale, the electronegativity of $\mathrm{H}$ and $\mathrm{C}$ is 2.20 and $2.55,{ }^{43}$ respectively. According to the Bader analysis, ${ }^{\mathbf{4 4}}$ the effective charges of $\mathrm{H}$ and $\mathrm{C}_{3}$ atoms are +0.074 e and -0.049 e, respectively. While shifting from the M-PG to WM-PG structure, the redistribution of charge density on PG is mainly induced by the on-site proton transfer. For both the M-PG and WM-PG systems, the charge of the chemisorbed hydrogen atom in PG is positive and less than $+1|e|$, just as that of the hydrogen atom in the processes of intermolecular proton transfer or intramolecular proton transfer.

Fig. 6 shows the computed STM image of the M-PG (Fig. 6(a) and (c)) and WM-PG (Fig. 6(b) and (d)) systems at a bias voltage of $-1 \mathrm{eV}$ (valence band). The tip in the STM images, Fig. 6(a) and (b), is set to be approximately $1.5 \AA$ lower than that of the third C layer in the PG sheet. The dark area in the STM image was located under the four-fold coordinated $\mathrm{C}$ atoms in the second layer of PG as if the $\mathrm{C}$ atom were absent because the four-fold coordinated $\mathrm{C}$ atoms layer were a little far from the point-like tip. Moreover, the three-fold coordinated $\mathrm{C}$ atoms in the third layer show bright spots because the charge spatial distribution of the $\mathrm{C}$ atoms near the Fermi level has a p-orbital character (Fig. $3\left(a_{1}\right)$ and $\left(b_{1}\right)$ ). The maximum height from the tip to the adsorbed $\mathrm{H}$ atom on the PG sheet in our calculations in the STM images is set to be about $0.5 \AA$, as shown in the Fig. 6(c) and (d). The STM images of the $\mathrm{H}$ atom adsorbed on the PG sheet show a bright spot centered on the $\mathrm{H}$ and $\mathrm{C}_{2}$ atoms due to the presence of the electronic states localized on the $\mathrm{H}$ and $\mathrm{C}_{2}$ atoms mainly in the valence band (Fig. $3\left(a_{1}\right)$ and $\left.\left(b_{1}\right)\right)$. The area of the bright spot of $\mathrm{H}$ atom in WM-PG is a little larger than that in the M-PG system. This fact indicates more charge at the $\mathrm{H}$ atom in the WM-PG system, which well conforms to the Bader charge analysis, as shown in Fig. 4(a) and (c).

\section{Conclusions}

In summary, an isolated hydrogen atom absorbed on PG sheet is predicted to induce magnetic moments and tune the electronic property. The larger binding energies and the negative formation energies suggest the stability of the M-PG and WMPG systems. The hydrogen adsorbed on the PG system can effectively tune the electronic properties of the PG sheet and change it from a semiconductor to half-metallic. Atomic $\mathrm{H}$ chemisorbed on the PG sheet on top of the $\mathrm{C}_{3}$ atom induces a magnetic moment due to the mixture of $\mathrm{sp}^{2}-\mathrm{sp}^{3}$ bonding and the magnetic coupling between $\pi$-electrons in the M-PG system. Through the on-site proton transfer, we can remarkably increase the magnetic moment of the PG sheets by 137 times. The results reveal that the energy difference between the M-PG and WM-PG systems is merely $0.03 \mathrm{eV}$. We believe that this method might allow us to develop magnetic nanoswitches to manipulate the magnetic state of the PG sheets at the atomicscale. We have also calculated the STM images for the adsorption of hydrogen on the PG sheet. The simulated STM images exhibit bright spots, depending strongly on the adsorbed hydrogen atom and the magnetic carbon atom. They are of great importance in future STM experiments for identifying the adsorption of hydrogen atom and the location of the magnetic carbon atom. Thus, our findings show that the ability to manipulate the magnetic state at the atomic-scale through onsite proton transfer on PG systems would have exciting applications in magnetic storage technology and next-generation electronic and spintronic nanodevices. Thus, we believe our design expands the diversity of PG applications.

\section{Conflicts of interest}

There are no conflicts to declare.

\section{Acknowledgements}

This work was supported by the National Natural Science Foundation of China (Grant No. 11474123) and the Natural Science Foundation of Jilin Province of China (Grant No. 20170101154JC). Lin-Lin Liu and Ying Wang contributed equally to this work.

\section{References}

1 H. Gonzalez-Herrero, J. M. Gomez-Rodriguez, P. Mallet, M. Moaied, J. Jose Palacios, C. Salgado, M. M. Ugeda, J.-Y. Veuillen, F. Yndurain and I. Brihuega, Science, 2016, 352, 437-441.

2 M. M. Monshi, S. M. Aghaei and I. Calizo, RSC Adv., 2017, 7, 18900-18908.

3 M. D. Xie, C. G. Tan, P. Zhou, J. G. Lin and L. Z. Sun, RSC Adv., 2017, 7, 20116-20122.

4 S. Zhang, J. Zhou, Q. Wang, X. Chen, Y. Kawazoe and P. Jena, Proc. Natl. Acad. Sci. U. S. A., 2015, 112, 2372-2377.

5 T. O. Wehling, M. I. Katsnelson and A. I. Lichtenstein, Phys. Rev. B: Condens. Matter Mater. Phys., 2009, 80, 085428. 
6 O. V. Yazyev, Phys. Rev. Lett., 2008, 101, 037203.

7 E. J. G. Santos, D. Sanchez-Portal and A. Ayuela, Phys. Rev. B: Condens. Matter Mater. Phys., 2010, 81, 125433.

8 H. Li, W. Chen, X. Shen, J. Liu, X. Huang and G. Yu, Phys. Chem. Chem. Phys., 2017, 19, 3694-3705.

9 K. Tao, P. Liu, Q. Guo, L. Shen, D. Xue, O. P. Polyakov and V. S. Stepanyuk, Phys. Chem. Chem. Phys., 2017, 19, 41254130.

10 X. Li, S. Zhang, F. Q. Wang, Y. Guo, J. Liu and Q. Wang, Phys. Chem. Chem. Phys., 2016, 18, 14191-14197.

11 T.-C. Wang, C.-H. Hsu, Z.-Q. Huang, F.-C. Chuang, W.-S. Su and G.-Y. Guo, Sci. Rep., 2016, 6, 039083.

12 J. Zhou, Q. Wang, Q. Sun, X. S. Chen, Y. Kawazoe and P. Jena, Nano Lett., 2009, 9, 3867-3870.

13 J. P. Perdew and Y. Wang, Phys. Rev. B: Condens. Matter Mater. Phys., 1992, 45, 13244-13249.

14 G. Kresse and J. Furthmüller, Phys. Rev. B: Condens. Matter Mater. Phys., 1996, 54, 11169-11186.

15 G. Kresse and D. Joubert, Phys. Rev. B: Condens. Matter Mater. Phys., 1999, 59, 1758-1775.

16 G. Makov and M. C. Payne, Phys. Rev. B: Condens. Matter Mater. Phys., 1995, 51, 4014-4022.

17 J. Neugebauer and M. Scheffler, Phys. Rev. B: Condens. Matter Mater. Phys., 1992, 46, 16067-16080.

18 F. Calle-Vallejo, J. Ignacio Martinez and J. Rossmeisl, Phys. Chem. Chem. Phys., 2011, 13, 15639-15643.

19 Y. Ding and Y. Wang, J. Mater. Chem. C, 2016, 4, 1517-1526.

20 J. Tersoff and D. R. Hamann, Phys. Rev. B: Condens. Matter Mater. Phys., 1985, 31, 805-813.

21 B. Zheng, P. Hermet and L. Henrard, ACS Nano, 2010, 4, 4165-4173.

22 H. Amara, S. Latil, V. Meunier, P. Lambin and J. C. Charlier, Phys. Rev. B: Condens. Matter Mater. Phys., 2007, 76, 115423.

23 Y. Fujimoto and S. Saito, Phys. Rev. B: Condens. Matter Mater. Phys., 2011, 84, 245446.

24 S. Okada, K. Nakada, K. Kuwabara, K. Daigoku and T. Kawai, Phys. Rev. B: Condens. Matter Mater. Phys., 2006, 74, 121412.
25 J. Lahiri, Y. Lin, P. Bozkurt, I. I. Oleynik and M. Batzill, Nat. Nanotechnol., 2010, 5, 326-329.

26 L. Kou, C. Tang, W. Guo and C. Chen, ACS Nano, 2011, 5, 1012-1017.

27 L.-L. Liu, C.-P. Chen, L.-S. Zhao, Y. Wang and X.-C. Wang, Carbon, 2017, 115, 773-780.

28 D. Yi, L. Yang, S. Xie and A. Saxena, RSC Adv., 2015, 5, 2061720622.

29 X. Chen, L. Xu, L.-L. Liu, L.-S. Zhao, C.-P. Chen, Y. Zhang and X.-C. Wang, Appl. Surf. Sci., 2017, 396, 1020-1025.

30 M. Z. S. Flores, P. A. S. Autreto, S. B. Legoas and D. S. Galvao, Nanotechnology, 2009, 20, 465704.

31 D. Cortes-Arriagada, S. Gutierrez-Oliva, B. Herrera, K. Soto and A. Toro-Labbe, J. Chem. Phys., 2014, 141, 134701.

32 Q. A. Cui and M. Karplus, J. Phys. Chem. B, 2002, 106, 79277947.

33 C. Tanner, C. Manca and S. Leutwyler, Science, 2003, 302, 1736-1739.

34 C. Fang, R. R. Frontiera, R. Tran and R. A. Mathies, Nature, 2009, 462, 200-204.

35 M. Pagacz-Kostrzewa, A. Bil and M. Wierzejewska, J. Photochem. Photobiol., A, 2017, 335, 124-129.

36 O. V. Yazyev and L. Helm, Phys. Rev. B: Condens. Matter Mater. Phys., 2007, 75, 125408.

37 L. Jeloaica and V. Sidis, Chem. Phys. Lett., 1999, 300, 157-162. 38 D. Soriano, N. Leconte, P. Ordejon, J.-C. Charlier, J.-J. Palacios and S. Roche, Phys. Rev. Lett., 2011, 107, 016602.

39 D. W. Boukhvalov, M. I. Katsnelson and A. I. Lichtenstein, Phys. Rev. B: Condens. Matter Mater. Phys., 2008, 77, 035427.

40 P. Esquinazi, R. Hohne, K. H. Han, A. Setzer, D. Spemann and T. Butz, Carbon, 2004, 42, 1213-1218.

41 P. O. Lehtinen, A. S. Foster, Y. C. Ma, A. V. Krasheninnikov and R. M. Nieminen, Phys. Rev. Lett., 2004, 93, 187202.

42 W. Tang, E. Sanville and G. Henkelman, J. Phys.: Condens. Matter, 2009, 21, 084204.

43 A. L. Allred, J. Inorg. Nucl. Chem., 1961, 17, 215-221.

44 G. Henkelman, A. Arnaldsson and H. Jónsson, Comput. Mater. Sci., 2006, 36, 354-360. 